\title{
Amplification and localization behaviors of obliquely incident light in randomly layered media
}

\author{
Wenji Deng \\ Department of Physics, South China University of Technology, Guangzhou 510641, People's Republic of China \\ and Department of Physics, Hong Kong University of Science and Technology, Clear Water Bay, Kowloon, Hong Kong \\ Zhao-Qing Zhang \\ Department of Physics, Hong Kong University of Science and Technology, Clear Water Bay, Kowloon, Hong Kong
}

(Received 23 October 1996; revised manuscript received 21 January 1997)

\begin{abstract}
We have extended the study of light amplification and localization behaviors in a randomly layered medium from normal to oblique incidence. Both $p$ and $s$ polarizations are considered. It is found that the statistical properties of the transmission and reflection coefficients depend only on $L / \xi_{0}(\theta)$ and $\xi_{0}(\theta) / l_{g}(\theta)$, independent of polarization. Here $L$ is the sample size, $\xi_{0}(\theta)$ is the localization length in the absence of gain, $\theta$ is the angle of incidence, and $l_{g}(\theta)=l_{g} \cos \theta$, where $l_{g}$ is the gain length. The localization length in the presence of gain is found to follow $1 / \xi(\theta)=1 / \xi_{0}(\theta)+1 / l_{g}(\theta)$. These scaling results reduce the problem from five parameters to two parameters and hold as long as the incident angle is below the critical angle of total internal reflection. A method of measuring $\xi_{0}(\theta)$ using resonant tunneling is proposed. Similar results are obtained for the case of dissipation. [S0163-1829(97)03822-8]
\end{abstract}

\section{INTRODUCTION}

It is well known that wave propagation in weakly disordered media can give rise to many interesting phenomena, such as coherent backscattering effects, speckle patterns, intensity-intensity correlation functions, and universal conductance fluctuations, etc., due to the interference of multiply scattered waves. ${ }^{1}$ Different from electrons, the electromagnetic wave can be coherently amplified or attenuated due to its bosonic nature. Therefore, the process of coherent amplification (or attenuation) can be described by a nonzero imaginary part of the dielectric constant of the medium. The interplay between disorder and amplification has been shown to give rise to many interesting new phenomena and is a subject under considerable studies recently both theoretically and experimentally. ${ }^{2}$ The sharpening of coherent backscattering cone in the presence of gain is an example. ${ }^{3-5}$ In one dimension (1D), the statistical properties of the reflection coefficient have been studied analytically by using a stochastic approach. ${ }^{6}$ By adopting a Gaussian white-noise model, it has been found in Ref. 6 that the probability distribution of the reflection coefficient depends only on two parameters, $\Lambda \equiv L / \xi_{0}$ and $q \equiv \xi_{0} / l_{g}$, where $L$ is the sample thickness, $\xi_{0}$ is the localization length in the absence of gain, and $l_{g}$ is the gain length, which is twice the inverse of gain coefficient. This two-parameter scaling result was later shown to hold also in the strong scattering and the short wavelength limit. ${ }^{7}$ It was also found in Ref. 7 that the localization length in the presence of gain, $\xi$, is reduced and follows $1 / \xi=1 / \xi_{0}$ $+1 / l_{g}$ due to the enhancement of the coherent backscattering effect. It is expected that the similar enhancement of the localization effect will occur in 2D and 3D systems in the strong scattering regime. It has been shown recently that, by using a dual symmetry between absorption and amplification, the localization length in an amplifying medium is the same as that in an absorbing medium, albeit the change of sign in the imaginary part of dielectric constant. ${ }^{8}$ The statis- tical distribution of the reflection coefficients for a multichannel system has also been studied recently in the weak scattering limit. ${ }^{9}$ In a multichannel system, each channel has different angles of incidence and channels are coupled by scatterings.

In this work, we have extended the above 1D study from normal incidence $(\theta=0)$ to oblique incidence $(\theta \neq 0)$. Both $p$ - and $s$-polarized (or $H$ - and $E$-polarized) waves are considered. Due to the existence of Brewster anomalies for the $p$-polarized wave, the localization length behaviors, $\xi_{0}(\theta)$, in the absence of gain are very different for $p$ - and $s$-polarized waves. ${ }^{10}$ For the case of $s$ polarization, $\xi_{0}(\theta)$ is a decreasing function of $\theta$. Whereas for the case of $p$ polarization, $\xi_{0}(\theta)$ is sharply peaked at Brewster's angle. The results of our study have shown that the two-parameter scaling results found earlier for the normal incidence can be generalized to the case of oblique incidence for both $p$ and $s$ polarizations by using the $\theta$-dependent localization length, $\xi_{0}(\theta)$, and gain length, $l_{g}(\theta)$ as long as the incident angle is below the critical angle of total internal reflection. These results can provide useful information for the study of multichannel systems in the strong scattering limit. ${ }^{12}$ The scaling reduces the problem from five parameters to two parameters and may provide a method of measuring $\xi_{0}(\theta)$ by using resonant tunneling spectrum. The model system, the Maxwell equations, and the transfer matrices for $p$ and $s$ polarizations will be constructed in Sec. II. Numerical results of scaling and localization length are shown in Sec. III, and conclusions will be given in Sec. IV.

\section{MODEL AND TRANSFER-MATRIX}

The system we considered is a randomly layered medium consisting of $L$ layers embedded in a homogenous medium with an effective dielectric constant $\epsilon_{0}$. Since the system is non magnetic, the magnetic permeability $\mu_{0}$ is taken to be unity here. Let $\Delta z_{n}$ be the thickness of the $n$th layer. $\Delta z_{n}$ is 
random and assumed to follow an exponential distribution $a^{-1} \exp \left(-\Delta z_{n} / a\right)$ with a mean thickness $a$, which is taken to be unity here. The dielectric constant in each layer can be written as $\varepsilon_{n}=\varepsilon_{n}^{\prime}-i \varepsilon^{\prime \prime}$. The real part is random from layer to layer, i.e., $\varepsilon_{n}^{\prime}=\varepsilon_{0}\left(1+\sigma \eta_{n}\right)$, where $0<\sigma<1$ gives the amplitude of randomness, and $\eta_{n}$ is a random number between $[-1,1]$. Since we are interested in the scaling properties of the amplified transmission and reflection coefficients and the effects on the localization length due to amplification, we assume the imaginary part $\varepsilon^{\prime \prime}$ to be a positive constant across the entire sample for simplicity. $\varepsilon^{\prime \prime}$ becomes negative if the medium is dissipative. In the absence of gain, the localization length behaviors of such a system have been studied both for normal ${ }^{11}$ and oblique incidences. ${ }^{10}$ For systems with gain (or dissipation), only the case of normal incidence has been considered so far. ${ }^{6,7,13}$ In this work, we consider the most general case of oblique incidence with gain (and dissipation) in the medium. We start from the following timeindependent Maxwell equations for the electric field in any given layer $n$ :

$$
\begin{gathered}
c^{2} \nabla^{2} \mathbf{E}_{n}(\mathbf{r})+\omega^{2} \varepsilon_{n} \mathbf{E}_{n}(\mathbf{r})=0, \\
\nabla \cdot \mathbf{E}_{n}(\mathbf{r})=0,
\end{gathered}
$$

where $\omega$ and $c$ are, respectively, the frequency and the speed of light in the vacuum. The magnetic field is related to the electric field by the equation $i \omega \mathbf{H}=c \nabla \times \mathbf{E}$.

Let $z$ be the layering direction and $x-z$ be the incident plane. For an incoming wave of wave vector $\mathbf{k}_{0}$ $=\left(k_{0 x}, 0, k_{0 z}\right)$, the continuity condition of electromagnetic wave at each interface requires $k_{n}^{(x)}=k_{0 x}$. Therefore, the complex wave vector inside the $n$th layer has the form $\mathbf{k}_{n}$ $=\left(k_{0 x}, 0, k_{n}^{(z)}\right)$ with $k_{n}^{(z)}=\beta_{n}-i \alpha_{n}$. From $\mathbf{k}_{n} \cdot \mathbf{E}_{n}=0$ and $c^{2} k_{n}^{2}=\omega^{2} \varepsilon_{n}$, we have

$$
\left(\frac{\beta_{n}}{k_{0}}\right)^{2}-\left(\frac{\alpha_{n}}{k_{0}}\right)^{2}=\frac{\varepsilon_{n}^{\prime}}{\varepsilon_{0}}-\sin ^{2} \theta
$$

$$
2\left(\frac{\beta_{n}}{k_{0}}\right)\left(\frac{\alpha_{n}}{k_{0}}\right)=\frac{\varepsilon_{n}^{\prime \prime}}{\varepsilon_{0}}
$$

where $\theta$ is the angle of incidence and $\sin ^{2} \theta=\left(k_{0 x} / k_{0}\right)^{2}$. The above equations give

$$
\begin{aligned}
\left(\frac{\beta_{n}}{k_{0}}\right)^{2}= & \frac{\varepsilon_{n}^{\prime}}{2 \varepsilon_{0}}\left[\sqrt{\left[1-\left(\varepsilon_{0} / \varepsilon_{n}^{\prime}\right) \sin ^{2} \theta\right]^{2}+\left(\varepsilon_{n}^{\prime \prime} / \varepsilon_{n}^{\prime}\right)^{2}}\right. \\
& \left.+\left(1-\frac{\varepsilon_{0}}{\varepsilon_{n}^{\prime}}\right) \sin ^{2} \theta\right], \\
\left(\frac{\alpha_{n}}{k_{0}}\right)^{2}= & \frac{\varepsilon_{n}^{\prime}}{2 \varepsilon_{0}}\left[\sqrt{\left[1-\left(\varepsilon_{0} / \varepsilon_{n}^{\prime}\right) \sin ^{2} \theta\right]^{2}+\left(\varepsilon_{n}^{\prime \prime} / \varepsilon_{n}^{\prime}\right)^{2}}\right. \\
& \left.-\left(1-\frac{\varepsilon_{0}}{\varepsilon_{n}^{\prime}}\right) \sin ^{2} \theta\right] .
\end{aligned}
$$

The general solution of electric field in the $n$th layer can be written as

$$
\mathbf{E}_{n}(\mathbf{r})=\left[\mathbf{a}_{n} e^{i k_{n}^{(z)}\left(z-z_{n}\right)}+\mathbf{b}_{n} e^{-i k_{n}^{(z)}\left(z-z_{n}\right)}\right] e^{i k_{0 x} x}
$$

where $z_{n}$ denotes the position of the interface between the $(n-1)$ th and $n$th layers. According to the directions of $\mathbf{a}_{n}$ and $\mathbf{b}_{n}$, the wave can be decomposed into the $p$ and $s$ polarizations. Below we consider these two cases separately.

(a) $p$-polarization: In this case, the electric field lies in the plane of incidence and $\mathbf{a}_{n}$ and $\mathbf{b}_{n}$ can be denoted by $\left(a_{n}^{(x)}, 0, a_{n}^{(z)}\right)$ and $\left(b_{n}^{(x)}, 0, b_{n}^{(z)}\right)$, respectively. By using the boundary conditions that both $E^{(x)}$ and $H^{(y)}$ are continuous at each interface, it is straightforward to show that the transfer matrix which relates the fields between two adjacent layers has the form

$$
\left(\begin{array}{c}
a_{n}^{(x)} \\
b_{n}^{(x)}
\end{array}\right)=\frac{1}{2}\left[\begin{array}{cc}
1 & 1 \\
1 & -1
\end{array}\right]\left[\begin{array}{cc}
e^{i k_{n-1}^{(z)} \Delta z_{n-1}} & e^{-i k_{n-1}^{(z)} \Delta z_{n-1}} \\
\frac{\varepsilon_{n-1}}{\varepsilon_{n}} \frac{k_{n}^{(z)}}{k_{n-1}^{(z)}} e^{i k_{n-1}^{(z)} \Delta z_{n-1}} & -\frac{\varepsilon_{n-1}}{\varepsilon_{n}} \frac{k_{n}^{(z)}}{k_{n-1}^{(z)}} e^{-i k_{n-1}^{(z)} \Delta z_{n-1}}
\end{array}\right]\left(\begin{array}{l}
a_{n-1}^{(x)} \\
b_{n-1}^{(x)}
\end{array}\right) \equiv M_{n, n-1}^{(p)}\left(\begin{array}{c}
a_{n-1}^{(x)} \\
b_{n-1}^{(x)}
\end{array}\right),
$$

where $\Delta z_{n-1}$ is the thickness of the $(n-1)$ th layer. For a sample of $L$ layers, the transfer-matrix relating the fields from one surface of the sample to the other surface becomes $M^{(p)} \equiv \Pi_{n=1}^{L+1} M_{n, n-1}^{(p)}$. If we denote $E_{t}, E_{r}$, and $E_{0}$ as the electric field amplitudes of the transmitted, reflected, and incident waves, respectively, i.e., $a_{L+1}^{(x)}=E_{t} \cos \theta, a_{0}^{(x)}=E_{0} \cos \theta, b_{L+1}^{(x)}=0$, and $b_{0}^{(x)}=E_{r} \cos \theta$, the reflection and transmission amplitudes can be obtained from the transfer matrix through

$$
r \equiv \frac{E_{r}}{E_{0}}=-\frac{M_{21}^{(p)}}{M_{22}^{(p)}} \quad \text { and } t \equiv \frac{E_{t}}{E_{0}}=\frac{\operatorname{det} M^{(p)}}{M_{22}^{(p)}}
$$

where $\operatorname{det} M^{(p)}$ and $M_{21}^{(p)}\left(\right.$ or $M_{22}^{(p)}$ ) are, respectively, the determinant and matrix element of $M^{(p)}$.

(b) $s$-polarization: In this case, the electric field is normal to the plane of incidence and has only $y$ component. The transfer matrix can be obtained from the continuity condition of $E^{(y)}$ and $H^{(x)}$ at each interface. Similar to the case of $p$ polarization, if we denote $E_{t}, E_{r}$, and $E_{0}$ as the electric field amplitudes of the transmitted, reflected, and incident waves, respectively, we find 


$$
\left(\begin{array}{c}
E_{t} \\
0
\end{array}\right)=M^{(s)}\left(\begin{array}{c}
E_{0} \\
E_{r}
\end{array}\right)
$$

where $M^{(s)} \equiv \Pi_{n=1}^{L+1} M_{n, n-1}^{(s)}$, and

$$
M_{n, n-1}^{(s)}=\frac{1}{2}\left[\begin{array}{cc}
\left(1+\frac{k_{n-1}^{(z)}}{k_{n}^{(z)}}\right) e^{i k_{n-1}^{(z)} \Delta z_{n-1}} & \left(1-\frac{k_{n-1}^{(z)}}{k_{n}^{(z)}}\right) e^{-i k_{n-1}^{(z)} \Delta z_{n-1}} \\
\left(1-\frac{k_{n-1}^{(z)}}{k_{n}^{(z)}}\right) e^{i k_{n-1}^{(z)} \Delta z_{n-1}} & \left(1+\frac{k_{n-1}^{(z)}}{k_{n}^{(z)}}\right) e^{-i k_{n-1}^{(z)} \Delta z_{n-1}}
\end{array}\right] .
$$

Thus, the reflection and transmission amplitudes become

$$
r \equiv \frac{E_{r}}{E_{0}}=-\frac{M_{21}^{(s)}}{M_{22}^{(s)}} \quad \text { and } t \equiv \frac{E_{t}}{E_{0}}=\frac{\operatorname{det} M^{(s)}}{M_{22}^{(s)}} .
$$

In both cases discussed above, the reflection coefficient, $R \equiv|r|^{2}$, and the transmission coefficient, $T \equiv|t|^{2}$, of each configuration of $\sigma$, depend on four parameters, i.e. $L, \omega, \varepsilon^{\prime \prime}$, and $\theta$. It is easy to show from Eq. (1) that they satisfy the following flux production relation:

$$
R+T=1+\frac{\omega}{c \cos \theta} \frac{\varepsilon^{\prime \prime}}{\sqrt{\varepsilon_{0}}} \int_{z_{1}}^{z_{L+1}} \frac{\mathbf{E} \cdot \mathbf{E}^{*}}{E_{0}^{2}} d z .
$$

The unitarity condition recovers when $\varepsilon^{\prime \prime}=0$.

\section{NUMERICAL RESULTS: SCALING AND LOCALIZATION LENGTH}

Before presenting our results for the case of oblique incidence, let us summarize briefly the scaling results found previously in the case of normal incidence. ${ }^{6,7}$ In the case of normal incidence $(\theta=0)$, it is easy to see that the transfer matrices in Eqs. (8) and (10) become identical. For any given set of medium parameters $\sigma$ and $\varepsilon^{\prime \prime}$, the random configuration of the dielectric constant in a sample of $L$ layers, i.e., $\varepsilon_{n}, n=1,2, \ldots, L$, is first generated according to the distribution described in Sec. II. The transmission $(T)$ and reflection $(R)$ coefficients can be calculated from Eqs. (9) and (12) at any frequency $\omega$. By taking the data of many configurations, we obtain a statistical distribution of the transmission (or reflection) coefficient. Thus, these distribution functions depend on four parameters, i.e., $\sigma, \varepsilon^{\prime \prime}, L$, and $\omega$. It was first shown in Ref. 6 that, in the long-wavelength limit, the probability distribution for the reflection coefficient depends only on two dimensionless variables $\Lambda \equiv L / \xi_{0}$ and $q \equiv \xi_{0} / l_{g}$, where $\xi_{0}$ is the localization length in the absence of gain and $l_{g}=2 C / \omega \varepsilon^{\prime \prime}$ is the gain length for the case of normal incidence. In the limit of infinite $\Lambda$, the distribution function of the reflection coefficient $R$ has the following analytic expression: ${ }^{6}$

$$
P_{\infty}\left(\frac{R-1}{2 q}\right)=\left(\frac{2 q}{R-1}\right)^{2} \exp \left(\frac{-2 q}{R-1}\right) .
$$

This two-parameter scaling behavior is later shown to hold also for the strong scattering and short wavelength limit. For instance, it was shown that for given values of $\Lambda$ and $q$, the distribution function for the reflection coefficient $R, P_{\Lambda}(R$ $-1 ; q)$, is function of $R-1$ only, independent of $\sigma, \varepsilon^{\prime \prime}, L$, and $\omega$ for a wide range of frequency and randomness. In the limit of large $\Lambda, P_{\Lambda}(R-1 ; q)$ converges to Eq. (14) and becomes a universal function with only one renormalized variable $(R-1) / 2 q$. This two-parameter scaling holds for the distribution of transmission coefficient as well. ${ }^{7}$ Here we are interested to know if this two-parameter scaling theory can be generalized to the case of oblique incidence, where the problem contains five parameters and the degeneracy of $p$ and $s$ polarization is lifted.

In the following, we present the results of extensive numerical calculations for the case of oblique incidence. The values of $\varepsilon_{0}$ and $c$ are set to be unity for convenience. For a given set of five parameters $\left(\sigma, \varepsilon^{\prime \prime}, L, \omega, \theta\right)$, the transmission $(T)$ and reflection coefficients $(R)$ are calculated for each given configuration according to Eqs. (9) and (12) for $p$ - and $s$-polarized waves, respectively. The distribution of $T$ and $R$ are obtained from the results of 10000 configurations in each case. Since the localization length in the absence of gain, $\xi_{0}(\theta)$, is one of the two relevant length scales of the problem, we first calculate this quantity according to $1 / \xi_{0}(\theta)=\langle-2 L / \ln [T(L)]\rangle$, where \langle\rangle denotes the configurational average and $L$ is between 5 and 10 times of $\xi_{0}(\theta)$. The parameters we have chosen for $\sigma$ and $\lambda(=2 \pi / \omega)$ are $(\sigma, \lambda)=(0.2,1),(0.3,5)$, and $(0.5,10)$. The localization length $\xi_{0}(\theta)$ is plotted as a function of $\theta$ in Fig. 1 for both $p$ - and $s$-polarized waves. The function $\xi_{0}(\theta)$ decreases monotonically for $s$ polarization but shows a sharp peak near $45^{\circ}$ for $p$ polarization. Because of these Brewster anomalies, the values of $\xi_{0}(\theta)$ can vary by three orders of magnitude as $\theta$ is varied. In the presence of gain, the gain length becomes the other relevant length scale of the problem. Along the layering direction, this length can be obtained from the inverse of $\alpha_{n}$ given in Eq. (6). To the lowest order, we find $l_{g}(\theta)$ $=\cos (\theta) l_{g}$ with $l_{g}=2 / \omega \varepsilon^{\prime \prime}$.

Our calculations were carried out in the following way. If the two-parameter scaling theory found in the case of normal incidence can be generalized to the case of oblique incidence, the two relevant parameters become $\Lambda \equiv L / \xi_{0}(\theta)$ and $q \equiv \xi_{0}(\theta) / l_{g}(\theta)$. For a given set of chosen $(\sigma, \lambda)$, we have picked $\theta=0^{\circ}, 10^{\circ}, 20^{\circ}, 35^{\circ}, 50^{\circ}$, and $60^{\circ}$ for both $p$ and $s$ polarizations. The values of $L$ and $\varepsilon^{\prime \prime}$ are set by the scaling parameters $\Lambda$ and $q$. Our calculations show that for any chosen values of $\Lambda$ and $q$ in the ranges of $0<\Lambda<10$ and $0.0005<q<10$, the probability distribution of $R$ (or $T$ ) falls into a single curve, independent of the values of $\sigma, \lambda, \theta, L$, 


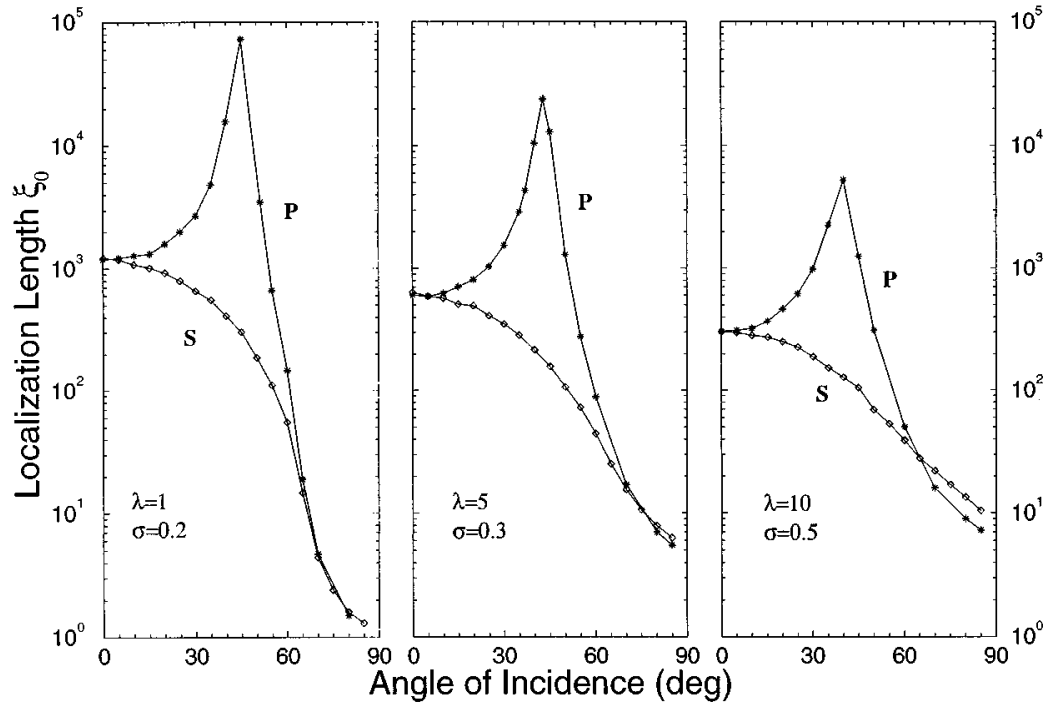

FIG. 1. In the absence of gain, the localization lengths $\xi_{0}(\theta)$ for both $p$ - and $s$-polarized waves are plotted at three different values of $\lambda$ and $\sigma$. $\varepsilon^{\prime \prime}$, and polarization. Since the case of $\theta=0$ is also included in our study, the distribution functions of $R$ and $T$ obtained here have the same functional forms as that found previously in the case of normal incidence. For instance, the explicit distribution functions for the case of $q=0.5$ and $\Lambda=0.3,0.6$ and 1.8 can be found in Figs. 1 and 2 of Ref. 7. This scaling holds as long as the incident angle is below the critical angle $\theta_{c}=\sin ^{-1} \sqrt{1-\sigma}$ of total internal reflection. When $\theta \geqslant \theta_{c}$, evanescent waves appear. In this case, the amplification of light due to multiple scattering is reduced and, therefore, produces a different distribution of $R$ (or $T$ ). Thus, by using the $\theta$-dependent localization length, $\xi_{0}(\theta)$, and gain length along the layering direction, $l_{g}(\theta)$, the scaling reduces a five-parameter problem to two parameters only and is independent of polarization.

Below the $\theta_{c}$, the distribution function of $R, P_{\Lambda}$, always saturates to the universal distribution Eq. (14) when $\Lambda$ is larger than some saturation length $\Lambda_{s}$. This saturation length can be determined from the standard deviation, $\Delta_{\Lambda}$, between the distributions $P_{\Lambda}$ and $P_{\infty} \cdot{ }^{7}$ We define $\Lambda_{s}$ as the value of $\Lambda$ where $\Delta_{\Lambda}$ is within $8 \%$ of its saturation value $\Delta_{\infty}$. In Fig.

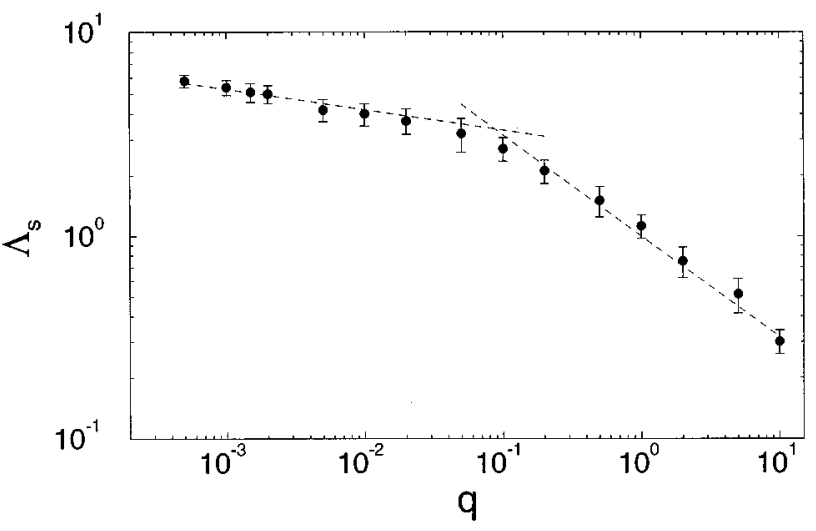

FIG. 2. The saturation length $\Lambda_{s}$ is plotted as a function of $q$. Two dashed lines show the function $2.65 q^{-0.1}$ for $q<0.1$ and $q^{-0.5}$ for $q>0.1$, respectively.
2, we plot $\Lambda_{s} \equiv L_{s} / \xi_{0}(\theta)$ as a function of $q \equiv \xi_{0}(\theta) / l_{g}(\theta)$ in $\log -\log$ scale. It should be pointed out that, besides the inclusion of $\theta \neq 0$, the range of scaling found here is much wider than that shown in Ref. 7. In Fig. 3, we show the distribution function $P_{\Lambda}[(R-1) / 2 q]$ at $\Lambda=\Lambda_{s}$ for 14 sets of randomly picked parameters $q, \lambda, \sigma, \theta$; four from $s$ polarization and ten from $p$ polarization. The values of $q$ goes from 0.0005 to 10 and the value of $\xi_{0}$ changes by $2-3$ orders of magnitude among these 14 sets. The shortest $\xi_{0} \approx 50$ comes from $s$ polarization with $q=0.05, \lambda=1, \sigma=0.2$, and $\theta=60^{\circ}$, while the longest $\xi_{0} \approx 12900$ comes from $p$ polarization with $q=5, \lambda=5, \sigma=0.3$, and $\theta=45^{\circ}$. The solid curve in Fig. 3 is the analytic result of Eq. (14). The collapse of all data points onto the solid line again demonstrates the validity of two-parameter scaling.

The saturation length shown in Fig. 2 has interesting physical implications. At large $q(0.1<q<10)$, the saturation length follows the relation $\Lambda_{s} \approx q^{-0.5}$, as shown by the dashed line. At the saturation length, the average value of $R$ diverges because the distribution function reaches $P_{\infty}$,

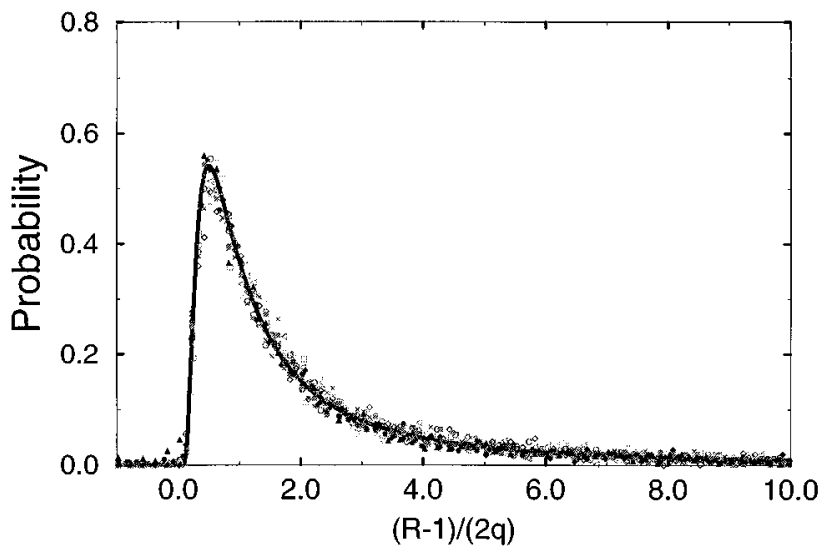

FIG. 3. The saturated probability distribution for the reflection coefficient $R$ is plotted as a function of renormalized variable ( $R$ $-1) / 2 q$ for 14 randomly picked sets of parameters for both $p$ and $s$ polarizations (see text). 
which is proportional to $1 / R^{2}$ for large $R{ }^{6}$ This implies that, at given values of $L, \sigma, \omega$, and $\theta$, some pole of the reflection (or transmission) coefficient can be reached with the presence of $l_{g}(\theta)$ for a particular configuration. In a complex frequency plane, this pole has a real part $\omega$ as the resonant frequency and an imaginary part $c / l_{g}$. Since the imaginary part of a pole is related to the width of the tunneling peak at resonance, i.e., $\Delta \omega \approx c / l_{g}$, the relation $L^{2} \approx\left[\xi_{0}(\theta) l_{g}(\theta)\right]$ found from saturation length curve in Fig. 2 now becomes

$$
\xi_{0}(\theta) \approx L^{2} \Delta \omega / \cos (\theta) c .
$$

Equation (15) actually relates the localization length of a particular resonant mode to the width of that mode, which can be measured from a tunneling spectrum. It also indicates that the transport of wave through resonant tunneling is diffusive even in one dimension. This new method of measuring the localization length has been successfully tested both theoretically and experimentally for the case of normal incidence. ${ }^{14}$ Our present study shows that this method of measuring the localization length of resonant states can be generalized to the case of oblique incidence as well. It should also be mentioned that this method applies when sample thickness is comparable to the localization length, i.e., 0.3 $<L / \xi_{0}(\theta)<3$, as can be seen from Fig. 2. In this region, the standard way of measuring the localization length, i.e., by measuring the decay length of $T(L)$, becomes inapplicable due to large fluctuations arising from resonances. ${ }^{15}$

As $q$ becomes smaller than $0.1, \Lambda_{s}$ tends to saturate to a constant value. This behavior can be understood by using the following arguments. In limit of small $q, \Lambda_{s}>4$ (or, $L$ $>4 \xi_{0}(\theta)$. The system is well inside the localized regime and the width of a resonant state becomes exponentially small. If we naively take those states localized near the center of the sample, we have $\Delta \omega \propto \exp \left(-L / 2 \xi_{0}\right) .^{15,16}$ By applying the scaling to the above relation, we expect a logarithmic dependence of $q$ on $\Lambda_{s}$ in the small $q$ regime. This is not inconsistent with the saturation behavior found in Fig. 2 in the small $q$ region, where the fitted dashed line for $q<0.02$ has the form $\Lambda_{s}=2.65 q^{-0.1}$.

Finally, we will discuss the localization length in the presence of gain. Here we will derive an analytic result for the case of $p$ polarization and compare it with the numerical simulations. The same result can be obtained for $s$ polarization. For a particular configuration " $i$," the flux production relation Eq. (13) relates the reflection, $R_{i}$, and transmission, $T_{i}$, coefficients to the electric field inside the sample. By substituting Eqs. (7) and the relations $a_{n}^{(z)}=-a_{n}^{(x)} k_{0 x} / k_{n}^{(z)}$ and $b_{n}^{(z)}=b_{n}^{(x)} k_{0 x} / k_{n}^{(z)}$ for the $p$ polarization into the RHS of Eq. (13), we find

$$
\int_{z_{1}}^{z_{L+1}} \frac{\mathbf{E} \cdot \mathbf{E}^{*}}{E_{0}^{2}} d z \approx \sum_{n=1}^{L}\left(1+\frac{k_{0 x}^{2}}{\alpha_{n}^{2}+\beta_{n}^{2}}\right) \frac{\left|a_{n}^{(x)}\right|^{2}+\left|b_{n}^{(x)}\right|^{2}}{E_{0}^{2}} \Delta z_{n} .
$$

In the above equation, we have ignored the cross terms. These terms contain a random phase and are assumed to vanish after summation. Using Eq. (3) and replacing the summation by integration in Eq. (16), we can write Eq. (13) as

$$
\begin{aligned}
R_{i}+T_{i} \approx & 1+\frac{\omega \varepsilon^{\prime \prime}}{c \sqrt{\varepsilon_{0}} \cos \theta}\left\{\frac{1}{\cos ^{2} \theta}+0\left[\left(\frac{\varepsilon^{\prime \prime}}{\cos ^{2} \theta}\right)^{2}\right]\right\} \\
& \times \int_{z_{1}}^{z_{L+1}} \frac{\left|a_{n}^{(x)}\right|^{2}+\left|b_{n}^{(x)}\right|^{2}}{E_{0}^{2}} d z .
\end{aligned}
$$

Assuming that the field amplitudes decay exponentially from the input surface with a localization length $\xi_{i}(\theta)$, we can write

$$
\begin{gathered}
\frac{\left|a_{n}^{(x)}\right|^{2}}{E_{0}^{2}} \simeq e^{-2\left(z-z_{1}\right) / \xi_{i}(\theta)} \cos ^{2} \theta, \\
\frac{\left|b_{n}^{(x)}\right|^{2}}{E_{0}^{2}} \simeq \mathrm{Re}^{-2\left(z-z_{1}\right) / \xi_{i}(\theta)} \cos ^{2} \theta .
\end{gathered}
$$

In the limit of large $L$, we have $T_{i}=0$. By neglecting higher order term in Eq. (17), we find

$$
\frac{1}{\xi_{i}(\theta)}=\frac{1}{l_{g}(\theta)}+\frac{2 q}{R_{i}-1} \frac{1}{\xi_{0}(\theta)},
$$

where we have used $q \equiv \xi_{0}(\theta) / l_{g}(\theta)$ and $l_{g}(\theta)=l_{g} \cos \theta$, with $l_{g}=2 c / \omega \varepsilon^{\prime \prime}$. By taking the configurational average on both sides of the above equation and using the distribution in Eq. (14), we finally obtain

$$
\frac{1}{\xi(\theta)}=\frac{1}{l_{g}(\theta)}+\frac{1}{\xi_{0}(\theta)} .
$$

The above equation is the generalization of the result obtained in Ref. 7 from normal to oblique incidence and is also true for $s$-polarized waves. The reduction of localization length in the presence of gain is due to the enhancement of coherent backscattering effect. For a given $l_{g}$, the reduction is more significant for $p$ polarization than $s$ polarization, particularly, near the Brewster anomalies. Equation (20) has been confirmed by our numerical simulations. The localization length $1 / \xi(L, \theta)=\left\langle 1 / \xi_{i}(L, \theta)\right\rangle=-\left\langle\ln \left[T_{i}(L, \theta)\right]\right\rangle / 2 L$ is calculated as a function of $L$. For all the cases we have studied, the function $\xi(L, \theta)$ approaches $\xi(\theta)$ of Eq. (20) in the large $L$ limit.

\section{CONCLUSIONS}

In summary, we have extended the two-parameter scaling theory in randomly layered media from normal to oblique incidence for angles below the critical angle of total internal reflection. The scaling holds for both $p$ - and $s$-polarized waves. The only two relevant lengths are the $\theta$-dependent localization length in the absence of gain, $\xi_{0}(\theta)$, and the gain length, $l_{g}(\theta)$, in the layering direction. The statistical properties of the transmission and reflection coefficients depend on two dimensionless parameters only, i.e., $L / \xi_{0}(\theta)$ and $\xi_{0}(\theta) / l_{g}(\theta)$. The localization length in the presence of gain is reduced and follows $1 / \xi(\theta)=1 / \xi_{0}(\theta)+1 / l_{g}(\theta)$. It should be mentioned that we have also studied the case of dissipation, where $\varepsilon^{\prime \prime}$ becomes negative. Like the case of normal incidence studied in Refs. 6 and 7, our present numerical simulations show that the above two-parameter scaling theory also applies to the case of dissipation if the gain 
length $l_{g}$ is replaced by the attenuation length $l_{a}=$ $-2 c / \omega \varepsilon^{\prime \prime}$. The distribution of $R$ remains the same as that of normal incidence and approaches the analytic form ${ }^{6}$

$$
P_{\infty}(R)=2 q \exp (2 q) \exp [-2 q /(1-R)] /(1-R)^{2},
$$

in the large $\Lambda$ limit. We have also found both analytically and numerically that the localization length follows Eq. (20) with $l_{g}(\theta)$ replaced by $l_{a} \cos \theta$.

Thus, we have shown that two-parameter scaling is quite robust in 1D. It holds for a wide range of frequency, randomness, and incident angle. The scaling brings the problem from five parameters to two only. It breaks down when total internal reflection occurs. In this case, the existence of eva- nescent waves alters the nature of wave propagation and makes the scaling invalid. The scaling results shown here indicate that the method of measuring the localization length using the resonant tunneling spectrum can be generalized from normal to oblique incidences. Although this work is limited to 1D study, the results obtained here for the case of oblique incidence can provide useful information for the study of localization length in an amplifying multi-channel system in the strong scattering limit. ${ }^{12}$

\section{ACKNOWLEDGMENTS}

This work was supported by Hong Kong RGC Grant No. HKUST 617/95P.
${ }^{1}$ For example, see Scattering and Localization of Classical Wave in Random Media, edited by P. Sheng (World Scientific, Singapore, 1990).

${ }^{2}$ For example, see M. Kempe, G. A. Berger, and A. Z. Genack, in Handbook of Optical Properties, edited by R. E. Hummel and P. Wibmann (CRC Press, Boca Raton, 1996), Vol. II.

${ }^{3}$ A. Yu. Zyuzin, Europhys. Lett. 26, 517 (1994).

${ }^{4}$ D. S. Wiersma, M. P. van Albada, and A. Lagendijk, Phys. Rev. Lett. 75, 1739 (1995).

${ }^{5}$ S. C. Feng and Z. Q. Zhang, Phys. Rev. B 54, 3240 (1996).

${ }^{6}$ P. Pradhan and N. Kumar, Phys. Rev. B 50, 9644 (1994).

${ }^{7}$ Z. Q. Zhang, Phys. Rev. B 52, 7960 (1995).

${ }^{8}$ J. C. Paasschens, T. Sh. Misirpashaev, and C. W. J. Beenakker, Phys. Rev. B 54, 11887 (1996).
${ }^{9}$ C. W. J. Beenakker, J. C. Paasschens, and P. W. Brouwer, Phys. Rev. Lett. 76, 1368 (1996).

${ }^{10}$ J. E. Sipe, P. Sheng, B. S. White, and M. H. Cohen, Phys. Rev. Lett. 60, 108 (1988).

${ }^{11}$ P. Sheng, B. White, Z. Q. Zhang, and G. Papanicolaou, Phys. Rev. B 34, 4757 (1986).

${ }^{12}$ C. C. Wong and Z. Q. Zhang (unpublished).

${ }^{13}$ A. R. McGurn, K. T. Christensen, F. M. Mueller, and A. A. Maradudin, Phys. Rev. B 47, 13120 (1993).

${ }^{14}$ Z. Q. Zhang, K. C. Chiu, and D. Zhang, Phys. Rev. B 54, 11891 (1996).

${ }^{15}$ W. Xue and P. A. Lee, Phys. Rev. B 38, 3919 (1988).

${ }^{16}$ Z. Q. Zhang and P. Sheng, Phys. Rev. B 44, 3304 (1991). 